OPINION

\title{
Ethics in mental health in resource poor setting: experiences from Nepal
}

\author{
Address: 1. Consultant Psychiatrist and former Director of Mental Hospital. *2. Psychiatrist, Mental Hospital \\ Email *Corresponding author
}

\begin{abstract}
Rapid advances in science and technology have contributed to an increased attention being given to medical ethics. Within the health sector questions are being asked about the possible threats to the accepted principles of equity and social justice in the delivery of care.

The article primarily highlights on the ethics in psychiatry. It brings into light a few shortcomings in current ethical issues in day to day psychiatric practice in Nepal and makes valid recommendations.

It concludes that in resource poor developing countries like Nepal, many factors including lack of resources, lack of skilled professionals and the absence of basic laws and regulations in the field of mental health, pose serious drawbacks which limit us from fully implementing standard ethical practices.
\end{abstract}

Key words: Medical ethics, psychiatry

\section{INTRODUCTION}

Simply speaking, ethics is the philosophical/moral tramework or theory that can be used to facilitate the discussion and decision-making process that leads to the resolution of the dilemma. The "moral framework" that is chosen for ethical decisions may be based either on the principles of Western bioethics (i.e., autonomy, beneficence, non-maleficence, justice), or on a human rights model, or an international law or a religious faith. ${ }^{24}$

Medical ethics is primarily a field of applied ethics, which is the study of moral values and judgments as they apply to the field of medicine. Some of the important values that commonly apply to medical ethics are:

- Beneficence - a practitioner should act in the best interest of the patient.

- Non-maleficence - "first, do no harm" - a practitioner should not do any harm to the patient

- Autonomy - the patient has the right to refuse or choose their treatment.

- Justice - concerns the distribution of scarce health resources in a rational manner, and the decision of who gets what treatment.

- Dignity - the patient (and the person treating the patient) have the right to dignity.

- Truthfulness and honesty

Values such as these do not give answers as to how to handle a particular situation, but provide a useful framework for understanding conflicts. ${ }^{2,48}$

Medical ethics has gained importance and urgency in the recent past due to a number of reasons. Rapid advances in science and technology, radical changes Upadhyaya et al: Ethics in mental health ... in macroeconomic planning and the introduction and promotion of market economies and globalization have all contributed to an increased attention being given to ethics, especially in health care. Within the health sector, questions are being asked about the possible threats to the accepted principles of equity and social justice in the delivery of care. In view of these developments, the Governing Bodies of WHO, both at the global and regional levels, have recommended that health ethics should be promoted through a number of activities, including research and teaching. ${ }^{5,8}$

\section{MEDICAL ETHICS IN THE NEPALESE CONTEXT}

\section{Historical aspects}

The history of modern medical practice in Nepal is relatively short. Prior to 1951, Nepal was under an autocratic regime, which kept a strict control over the introduction of modern education and health facilities into the country. There were very few health institutions and the number of qualified doctors was around 20 in 1951, which year marked the onset of democracy in Nepal. ${ }^{12}$

The association of medical doctors came into existence ahead of a medical council. The Nepal Medical Association, in the year 1962, demanded the establishment of a medical council to regulate the medical practice and during its first national conference in 1963, it unanimously passed a resolution demanding the creation of the Nepal Medical Council. Subsequently, the Nepal Medical Council Act was enacted in 1964. Though the Act 
was passed in 1964, it took another two years before the provisions of the Act were implemented. ${ }^{12}$ The Medical Council Act laid down some guidelines regulating the medical practice in Nepal. Prior to the enactment of the Act, medical practice was a matter of law and order and was regulated by the provisions of the National Civil Code (Muluki Ain). The earliest legislation in relation to medical practice can be traced to the publication of "Ilaj garne ko" (on treatment) in Muluki Ain (Law of the land) in 1853. 12

Since the establishment of the Nepal Medical Council in 1966, it has been made mandatory that the doctors wishing to work in Nepal should register themselves with the Council and sign an oath to follow the code of ethics developed by it. ${ }^{12}$

However, the Nepal Medical Council is a regulatory body with inadequate infrastructure to instruct and educate the doctors and to supervise their work. Similarly, it has no mechanism to help doctors to resolve the ethical dilemmas they may face in their day-to-day practice. ${ }^{12}$

\section{Ethics in clinical practice}

The NMC Code of Ethics guides medical practitioners regarding behavior in their general clinical practice. The Code of Ethics is divided into different sections. Each section deals with a specific issue. The issues dealt with in the code are: general principles; duties of physicians to their patients; duties of physicians to the profession; duties of a physician to another physician; duties of physicians in consultation, and disciplinary action. However, there are no special ethical codes which govern practice in specialized health sectors, and thus, there is no separate code of ethics in the field of mental health. ${ }^{12}$

\section{Research ethics}

Research in health is relatively new in Nepal.. Modern research in health started when the Nepal Health Survey was conducted in 1966. Most of the research studies in Nepal has been descriptive in nature. It is only recently that research, with experimental design involving human subjects is also being conducted. These developments prompted the government to establish a Medical Research Council in the country. ${ }^{12}$

Initially a committee named as the Nepal Medical Research Council (NMRC) was established in 1982 within the Ministry of Health under the chairmanship of the Health Secretary. This was to be the national focal point on health services research. The NMRC later formed an Ethical Review Board which was given the task of reviewing research proposals from an ethical point of view. ${ }^{12}$

Subsequently, the Nepal Medical Research Council (NMRC) was substituted by the Nepal Health Research Council (NHRC), an autonomous body established by an Act of Parliament in 1991. The NHRC has been made responsible for granting permission to research proposals. The NHRC is responsible for looking into the ethical aspects of research. The guidelines laid by the Council for International Organizations of Medical Sciences (CIOMS) are used by the NHRC as reference documents for considering the ethical issues in any research activity. ${ }^{12}$

The NHRC executive committee has a standing Technical Review Committee which scrutinizes all applications for research. In many cases a research proposal is approved by this committee itself, but if there are any ethical issues which need further consideration, the proposal is referred to the Ethics Subcommittee. The Ethics Subcommittee regulates the ethical aspects of any research proposal submitted to it for approval. All research activities related to health have to be approved by the NHRC. ${ }^{12}$

\section{ETHICS IN PSYCHIATRY}

\section{Common ethical issues in medicine and psychiatry}

In the past few decades psychiatric units have been established in general hospitals as well as in medical colleges / academic institutions and in the private settings. This has brought the practice of psychiatry closer to that of general medicine but as a result, psychiatry is also facing many ethical issues common to several other medical specialties. These include - commercialization, an exaggerated emphasis on unnecessary laboratory investigations and technical procedures, increasing use of self-advertisement, 'cut backs' for investigations or services, fee splitting and an unhealthy relationship with pharmaceutical firms. These are unethical practices seen in every other branch of medicine including psychiatry ${ }^{6,7}$ The general code of ethics set by the Nepal Medical council helps in regulating such type of unethical practices and there are some penalties and punishments prescribed for violation of these types of ethical matters.

\section{Some ethical issues peculiar to psychiatry}

The practice of psychiatry is different from other medical specialties in two significant respects. Firstly, in psychiatry, one deals with certain groups of patients whose judgment may be impaired at times due to their mental illness or who are unable to look after themselves. Such patients, at times, may also become a danger to either themselves or others but may still refuse any medical help. In such situations, therapeutic intervention or even detention in a psychiatric facility against the patient's wishes may become necessary. This raises various ethical and human rights issues that have been debated extensively without arriving at a consensus. ${ }^{10}$

Second, in no other medical specialty do patients share with their doctor so many intimate details about their personal, emotional, social or even sexual life. As a result, a special kind of relationship, both 
positive and negative, develops between the patient and psychiatrist. This particularly happens during prolonged treatment. This raises many ethical issues depending on how the psychiatrist handles it. ${ }^{10}$

The psychiatric practices, akin to any other medical practice, involve ethical considerations. Psychiatrists have the duty to be aware of the specific ethical needs of psychiatry.

However, psychiatry involves ethical dilemmas in defining normal and abnormal, diagnosis and treatment, individual freedom, confidentiality, competency, commitment and other complex issues. Some of these ethical guiding principles change with time and the place of practice. ${ }^{10,11}$

Systematic understanding of psychiatric ethics is of recent origin. The American Psychiatric Association appointed a committee to develop a code of ethics only in 1970. The World Psychiatric Association developed a code of ethics in 1977. In our own neighborhood, a panel was appointed by the Indian Psychiatric Society to prepare the code of ethics for psychiatrists in India. The code was approved at its annual conference in Cuttack in 1989. ${ }^{6,10}$

Internationally, the ethical principles that govern the practice of psychiatry have been enlisted in the 'The Declaration of Madrid'. It includes guidelines that focus on the aims of psychiatry to treat mentally ill patients, prevent mental illness, promote mental health and provide care and rehabilitation for patients. The World Psychiatric Association (WPA) also approved these ethical standards that should govern the conduct of psychiatrists worldwide. Each patient has the right to know it and check if his/her psychiatrist respects this code ${ }^{1}$

\section{Madrid Declaration on Ethical Standards for Psychiatric Practice (August 2002) ${ }^{1}$}

- Psychiatry is a medical discipline concerned with the provision of the best treatment for mental disorders, with the rehabilitation of individuals suffering from mental illness and with the promotion of mental health.

- Psychiatrists should serve patients by providing the best therapy available consistent with accepted scientific knowledge and ethical principles.

- Psychiatrists should devise therapeutic interventions that are least restrictive to the freedom of the patient and seek advice in areas of their work about which they do not have primary expertise. While doing so, psychiatrists should be aware of and concerned with the equitable allocation of health resources.

- It is the duty of psychiatrists to keep abreast scientific developments of the specialty and to convey updated knowledge to others. Psychiatrists trained in research should seek to advance the scientific frontiers of psychiatry.

Upadhyaya et al: Ethics in mental health ...
- The patient should be accepted as a partner by right in the therapeutic process. The psychiatristpatient relationship must be based on mutual trust and respect to allow the patient to make free and informed decisions. It is the duty of psychiatrists to provide the patient with relevant information so as to empower the patient to come to a rational decision according to personal values and preferences.

- When the patient is incapacitated and/or unable to exercise proper judgment because of a mental disorder, or gravely disabled or incompetent, the psychiatrists should consult with the family and, if appropriate, seek legal counsel, to safeguard the human dignity and the legal rights of the patient.

- No treatment should be provided against the patient's will, unless withholding treatment would endanger the life of the patient and/or those who surround him or her. Treatment must always be in the best interest of the patient.

- Information obtained in the therapeutic relationship should be kept in confidence and used, only and exclusively, for the purpose of improving the mental health of the patient. Psychiatrists are prohibited from making use of such information for personal reasons, or financial or academic benefits. Breach of confidentiality may only be appropriate when serious physical or mental harm to the patient or to a third person would ensue if confidentiality were maintained. In these circumstances, psychiatrists, should whenever possible, first advise the patient about the action to be taken.

- Research that is not conducted in accordance with the canons of science is unethical. Research activities should be approved by an appropriately constituted ethical committee. Psychiatrists should follow national and international rules for the conduct of research. Only individuals properly trained for research should undertake or direct it. Because psychiatric patients are particularly vulnerable research subjects, extra caution should be taken to safeguard their autonomy as well as their mental and physical integrity. Ethical standards should also be applied in the selection of population groups, in all types of research including epidemiological and sociological studies and in collaborative research involving other disciplines or several investigating centers

The essence of all these recommendations is that psychiatric patients should be treated with dignity and respect. As far as possible, their informed consent must be taken for any treatment or hospital admission. If such patients are not in a position to give their consent, close family members should be consulted, but the interest of the patient must remain paramount. Physical restraints, if required, must be 
minimum and for a temporary period under close medical supervision. The use of chains or other degrading devices to restrict the patient should have no place in modern psychiatry. The patient should be kept as involuntary admission in a psychiatric hospital for the minimum period necessary. There must be adequate provisions for the right to appeal against forcible detention.

The patient should receive the best possible treatment which should be provided on the basis of recent and updated knowledge.

Attention to psychiatric ethics is one way we can advance what is good for our patients, our profession, and our society. Attention to the issue of ethics for the practice of psychiatry is increasing, in both the developed and the developing countries of the world.

\section{ETHICS IN MENTAL HEALTH IN NEPAL}

There is considerable variation in the extent to which ethical codes are promulgated in the different countries. For example, in the Scandinavian countries the medical profession has a long tradition of advancing human rights, so strong ethical codes are in place and medical ethics is taught in all Scandinavian medical schools. ${ }^{3}$

However, in resource poor developing countries like Nepal, one must face the reality of poor allocation of resources to health care and especially to psychiatry. Thus, it is difficult to adhere to the ethical guideline of providing the best treatment available for mental illness, including rehabilitation, as called for by the Declaration of Madrid.

Mental disorders account for nearly $12.3 \%$ of the global disease burden, estimated to increase to $15 \%$ by the year 2020. Much of this increase is likely to happen in developing countries that are least prepared to deal with it. In Nepal, the rough prevalence of mental illnesses is estimated to be around $25-30 \%$ of the total population. Mentally ill individuals who live in rural communities have significant health care needs but may experience many obstacles to obtaining adequate health care services. Especially in the more remote areas, barriers to care include insufficient access to multidisciplinary clinicians, emergency psychiatric services, mental health clinics, mental hospitals, and innovative psychotropic medicines and other therapies. More basic community services, such as transportation, electricity, water, and communication systems, that are important to the provision of optimal medical care also may not be available in many of these areas. ${ }^{9}$

Due to paucity of financial resources and the time taken to train professionals, developing countries like Nepal are unlikely to see substantial short-term increases in the number of mental health professionals. Although effective interventions for many mental disorders are available, only a minority

Upadhyaya et al: Ethics in mental health ... receive treatment from mental health services. Many of the primary-care clinicians / paramedics are either unaware or wary of modern psychotropic drugs.

Important but little-recognized ethical problems do arise in health care, and these dilemmas may greatly influence how mental health services are delivered by caregivers and experienced by patients. These issues merit greater attention to enhance our understanding and implementation of psychiatric care services. ${ }^{9}$

A few shortcomings in current ethical issues in day to day psychiatric practice in Nepal can be attributed to:

\section{Lack of basic laws and regulations governing Mental Health: Absence of a mental health act, any National Mental health program and any special code of ethics for mental health professionals in Nepal poses a serious drawback for implementing the ethical guidelines as prescribed by the Madrid declaration. There are almost no laws which safeguard the rights of a mentally ill patient.}

2. Lack of adequate mental health services: $A$ majority of the total 75 districts of Nepal do not have any mental health care facility. Thus, a large proportion of the people in need of mental health care services are not receiving any appropriate services. There is gross lack of human resources providing different types of mental health services. Thus, it is virtually not possible to provide the best available treatments for mental disorders. Similarly, the rehabilitation of individuals suffering from mental illness and the activities aimed at promotion of mental health are also grossly inadequate and are not keeping in with the international standards of basic mental health care.

Caregivers face serious clinical ethical dilemmas every day. Because of poor resources, clinicians / paramedics commonly provide care without optimal supports, services, and safeguards for their patients. Health care providers find it necessary at times to ration care, to provide care outside of their usual areas of expertise and competence, to deal with patients' "noncompliance" related to access problems, and to make complex clinical decisions without the benefit of specialists consultations.

Care for patients with mental illnesses in resource poor settings poses further problems, such as addressing patients' potential for self-harm and violence, dealing with the heightened social stigma associated with mental disorders, protecting vulnerable patients from potential abuse or exploitation, and grappling with care planning for individuals with impaired decision-making capacity. These ethically rigorous issues are often more acute in health care settings of Nepal, primarily because usual practices to ensure ethical conduct are not possible because of the scarcity of health care resources. 


\section{Cultural issues}

Experiences of suffering, definitions of illness, and care-seeking behaviors are influenced by cultural values and beliefs. Particularly cultural ethical "errors" may occur through underinterpretation or overinterpretation of the cultural meanings of certain symptoms, signs, complaints, or behaviors. Such errors may greatly interfere with mental health care of patients and their families in the future.

Sensitivity and acumen about the cultural dimensions of mental health care are critical in a developing country like Nepal, as the culture based and religious beliefs / explanations of mental illnesses are very much prevalent in the society. Ethical and rational practice in treating mentally ill patients is essential, but it should strike a balance with the cultural belief systems of the patient.

\section{4. "Generalist" care and multidisciplinary team issues}

Multidisciplinary approaches to mental health care are recognized as beneficial and practical because strengths from various professional specialty fields may be combined to implement more robust, comprehensive care. However, in Nepal, "generalist" care is the rule. Generalist care is characterized by individuals without specialty training who function in expanded roles to care for complex, multiproblem patients. Primary care professionals, including paramedics and even nonprofessionals provide treatment in rural areas but often without the support or backup of specialists in mental health.

These generalist issues have critical ethical ramifications because working outside of one's sphere of competence in mental health care, except in emergencies, essentially violates ethical norms for the profession of medicine and related fields. This may lead to serious mismanagement, including polypharmacy, prescribing medications without any working diagnoses or prescribing medications with abuse/dependence potential (like benzodiazepines), without any rational basis.

\section{Limited consultation for ethics issues}

Sound ethical care in complex cases at times requires a consultative process, with a bioethics committee, an ethics or legal expert, or a knowledgeable colleague. A crucial skill employed by clinicians to prevent ethical mistakes is the use of collaboration, consultation, and referral. However, ethics review processes, both formal and informal, are not available in Nepalese health care settings. Ethics committees do not exist. Furthermore, ethics experts and specialist colleagues, peers, and supervisors are mostly not available. These factors place the mental health practitioner at risk for true ethical misjudgments.

Upadhyaya et al: Ethics in mental health ...

\section{J Psychiatric Association of Nepal Vol.1, No.1, 2011}

\section{Research / Treatment on patients with mental} disorders

In general, it is agreed that for any medical research on human beings, informed consent of the individual must be an essential part of the research protocol. The difficulty in seriously mentally ill patients is that due to their illness, many of them have their judgement substantially impaired. They may not be in a position to judge the risks involved in various medical research procedures. In this case, a an informed consent from the patient's caregivers / guardians is essential.

However, in Nepal, where a large number of patients are poorly educated, giving consent by signing some research protocol or after reading some treatment modality seems to be an inadequate safeguard. The patients and their families inherently trust their doctors and hence a big ethical responsibility falls on the treating doctor. A complete ban on all research on the mentally ill may be going to one extreme, but this is not plausible. To protect against any abuse, such research should be strictly limited to what is in the larger interest of the mentally ill. Second, there must be an independent monitoring to ensure that ethical guidelines are followed.

\section{The question of electroconvulsive therapy (ECT)}

The use of unmodified ECT is another subject of ethical debate in a country like Nepal.

Though the use of unmodified ECT is considered ethically unsound, there are patients who will require ECT, and it may even be life saving in some situations. It must be ensured that ECT is provided with anaesthesia and muscle relaxants, wherever such facilities are available. But since ECT itself is not available except in few setups in Nepal, and the availability of anaesthesia and muscle relaxants is much scarce, thus, the use of direct ECT can't be called totally unethical in the Nepalese setting.

\section{Lack of awareness about ethical rights:}

Consumers of mental health services, in a developing country like Nepal, are not aware of their ethical rights in relation to mental health. This makes them more vulnerable to ethical misconducts and violations of their rights.

\section{Mental health care for all:}

The most important national ethical issue in a country like Nepal is how to ensure that the benefit of modern psychiatry is available to all sections of our population. Unfortunately, it is not happening at present. Poor patients from rural areas or many in the urban areas also, have got little benefit from modern mental health care. The homeless mentally ill or the wandering 'lunatic' or mentally ill with torn clothes, with nothing to eat, ridiculed by passers-by is a common sight in many of the rural / urban areas. 
Similarly, because of the underlying stigma, majority of the patients still don't seek any help from mental health professionals.

\section{RECOMMENDATIONS}

The following approaches can be devised to ensure ethical practice in mental health in resource poor settings like Nepal:

(a) As the financial resources and trained professional are scarce, integration of the mental health care service with the existing general health services may be a good approach to provide at least the basic mental health services to all.

(b) The existing infrastructure of health services can be utilized to the maximum extent possible with the aim to deliver the minimum mental health care services;

(c) Appropriate task oriented training in mental health can be provided to the existing health staff;

(d) Mental health services can be incorporated into the existing community health programme. The design of the programme should be such that we make use of the existing infrastructure and personnel, ensuring that no extra expenditure is incurred in implementing the mental health programme. The medical practitioners can be 'trained' to address mental health issues also, and also the Primary health center staffers.

(e) The treatments that are provided must be culture friendly, that is, they should take the local cultural ethics into consideration, so that it is more readily acceptable.

(f) There should be prompt enactment and implementation of the mental health act and a National mental health program. Similarly, code of ethics for mental health professionals can be devised. Mental health professionals, or for that matter, even other health professionals coming in contact with the mentally ill patients must be made aware of the ethical principles guiding mental health practices and the rights of a mentally ill patient.

The issue of ethical principles guiding mental health practices for non - mental health professionals and other service providers coming in contact with the mentally ill patients is important, because people suffering from mental disorders are seen in Nepal by faith healers, general medical practitioners, and different specialists. They are also treated by alternative system of medicine like Ayurveda, Homeopathy and Unani system of medicine. Thus, ethical care for mental health professionals alone may not serve the purpose

(g) Raising the awareness of the general public about issues of ethical rights of consumers would be beneficial.

(h) There is a need for advocacy about the ethical issues related to mental health by mental health professionals. As mental health professionals are the expert in their field, it is their responsibility to maintain the standards of ethical practice in mental health.

\section{CONCLUSION:}

Ethical practice is essential in the present day world in the field of medicine, including psychiatry. The psychiatric ethics are guided by several codes of ethics which are accepted internationally. However, in resource poor developing countries like Nepal, many factors including lack of resources, lack of skilled professionals and the absence of basic laws and regulations in the field of mental health, pose serious drawbacks which limit us from fully implementing standard ethical practices.

\section{REFERENCES:}

1. The Madrid Declaration on Ethical Standards for Psychiatric Practice 2002.

2. Singer P A, Benatar S R. Beyond Helsinki: a vision for global health ethics - Brief Article - Editorial. British Medical Journal, 2001.

3. Gaw A C. Ethics, Culture, and Psychiatry: International Perspectives (edited by Ahmed Okasha, Julio Arboleda-Flórez, and Norman Sartorius), Book Review In Psychiatric Services 2001; 52:1111.

4. Pathare S. A mental health manual for primary care. Indian Journal of Medical Ethics, 2004; 12(4).

5. Desai N G. Responsibility of psychiatrists: Need for pragmatic idealism - Editorial. Indian Journal of Psychiatry, 2006; 48: 211-214.

6. Rao T. S. S. Ethics in psychiatry. Indian Journal of Medical Ethics, 1995; 3(3).

7. Lolas F. Ethics in psychiatry: a framework In World Psychiatry. 2006; 5(3): 185-187.

8. Ledermann E K. Ethics in psychiatry - the patient's freedom and bondage. Journal of Medical Ethic. 8: 191-194.

9. Roberts L W, Battaglia J and Epstein R S. Frontier Ethics: Mental Health Care Needs and Ethical Dilemmas in Rural Communities, Psychiatric Services 1999; 50:497-503.

10. Wig N N. Ethical issues in psychiatry. Indian Journal of Medical Ethics, Jul-Sep2004;12(3).

11. Davar B. Medical ethics in the context of the national mental health programme Indian Journal of Medical Ethics Jul-Sep1996; 4(3).

12. Adhikari R. K. Medical ethics in the Nepalese context (edited by Nimal Kasturiaratchi, Reidar Lie and Jens Seeberg), In Health ethics in six SEAR countries, Vol. 1, World Health Organization, Regional Office for South-East Asia. 